\title{
Effect of wall materials on the spray drying efficiency, powder properties and stability of bioactive compounds in tamarillo juice microencapsulation
}

\begin{abstract}
Hydro- and lipo-soluble bioactive compounds, such as anthocyanins and carotenoids, in tamarillo juice were microencapsulated using different wall materials, such as maltodextrin (MD), n-octenyl succinic anhydride modified starch, from waxy maize for high load encapsulation (OSA 1), low viscosity gum Arabic alternative (OSA 2), resistant maltodextrin (RMD) and gum Arabic (GA). The wall materials were characterized according to their physicochemical and functional properties, molecular weight distribution and encapsulation efficiency using X-ray diffractometry. The tamarillo powders obtained after spray drying were evaluated for their physicochemical and thermal properties, phenolic content, flavonoid content, antioxidant capacity and storage stability. Although there were significant differences in terms of the encapsulation efficiencies of the wall materials, yield, physical properties and storage stability of the spray dried powders, all of the wall materials successfully encapsulated the hydro- and lipo-soluble bioactive compounds. The viscosity, amorphous region and molecular weight of the wall material had positive influences on the encapsulation efficiency, powder properties and storage stability of the encapsulated tamarillo juice. The storage stability of the powders depended on their water activity, hygroscopicity and glass transition temperature $(\mathrm{Tg})$. The tamarillo powders showed greater anthocyanin and carotenoid degradation in the presence of light at $25{ }^{\circ} \mathrm{C}$ compared to the powders stored in the dark at $4{ }^{\circ} \mathrm{C}$. GA and OSA 1 resulted in the highest encapsulation efficiency for both the hydro- and lipo-soluble bioactive compounds, while OSA 1 and MSB showed the greatest storage stability. Reductions in the antioxidant activity, phenolic content and flavonoid content during storage will contribute to the degradation of anthocyanins and carotenoids.
\end{abstract}

Keyword: Microencapsulation; Tamarillo; Wall material; Anthocyanins; Carotenoids 